\title{
Reliability of familiarity rating of ordinary Japanese words for different years and places
}

\author{
Shigeaki Amano, Kaname Kasahara, and Tadahisa Kondo \\ NTT Corporation, Kyoto, Japan
}

\begin{abstract}
Two-word familiarity sets were measured in different years (1995 and 2002) and places (Kanto and Kinki, in Japan) for a large number of Japanese words, to examine the reliability of familiarity ratings. The correlation between the word familiarities of the two sets was extremely high $(r=.958, N=10,515)$. It is suggested that familiarity rating, at least for ordinary words found in a dictionary, is very reliable and not greatly affected by differences in years and places.
\end{abstract}

Word familiarity (hereafter, familiarity rating) is one of the most powerful variables for explaining the efficiency and accuracy of language and cognitive processing. Many previous studies have consistently revealed the strong effects of familiarity ratings on, for instance, lexical decision latency (Amano \& Kondo, 1999; Kreuz, 1987), naming latency (Amano \& Kondo, 1999; Connine, Mullennix, Shernoff, \& Yelen, 1990), semantic categorization latency (Lewellen, Goldinger, Pisoni, \& Greene, 1993), recognition score (Amano \& Kondo, 1999; Shulman, 1976), and recall score (Shulman, 1976). These latencies become shorter and the scores improve as the familiarity rating of a word increases.

One concern of previous studies has been the modality dependency of the familiarity rating. Pisoni and Garber (1990) reported that the correlation coefficient of the familiarity rating is over $.92(N=450)$ between an auditorily presented word (i.e., a spoken word; hereafter, an auditory word) and a visually presented word (i.e., a written word; hereafter, a visual word). They suggested that familiarity ratings are modality independent.

Amano, Kondo, and Kakehi (1995) measured familiarity ratings for about 62,000 auditory and visual words. They found that, when homophones and homographs were excluded, the correlation coefficient of the familiarity rating was high $(r=.808, N=31,702)$ between auditory words and visual words, but it was lower than that reported by Pisoni and Garber (1990). They suggested that the lower correlation coefficient was caused by Japanese orthography and that the familiarity rating is to some degree modality dependent.

As an extension of the study undertaken by Amano et al. (1995), Amano and Kondo (1999) measured familiarity ratings for about 88,000 words, which constitute all the word entries in the Shin-Meikai Japanese dictionary (4th ed., Kindaichi, Shibata, Yamada, \& Yamada, 1989). In their study, the words were presented not only audito- rily or visually but also auditorily-visually (i.e., a spoken word and a written word are presented simultaneously; hereafter, an audiovisual word). These three kinds of familiarity ratings are registered in a word-familiarity database, which is one of a series of Japanese psycholinguistic databases developed by Amano and Kondo (1999, 2000a) and Sakuma et al. (2005). Amano and Kondo (1999) reported that the correlation coefficients of the familiarity ratings $(N=88,490)$ were .726 between auditory and visual words, .775 between auditory and audiovisual words, and .947 between visual and audiovisual words when homophones and homographs were included in the calculation. When homophones and homographs were excluded, the correlation coefficients of the familiarity ratings $(N=34,901)$ were .899 between auditory and visual words, .922 between auditory and audiovisual words, and .980 between visual and audiovisual words. These results show that the familiarity ratings of auditory, visual, and audiovisual words have a high correlation. Their results suggested that familiarity ratings are to some degree modality dependent, but they are independent with regard to nonhomophones and nonhomographs.

Familiarity rating has been the subject of many studies, as described above. However, little attention has been paid to its reliability. Because a familiarity rating is a subjective variable, it is probably affected by many factors related to the rater's experience and knowledge of the words to be rated. It is suspected that familiarity rating might be inconsistent, for example, across year and place, thus making it an unreliable variable.

The present study investigated whether familiarity rating is reliable for different years and places, using over 10,000 test words. Two sets of familiarity ratings were compared in this study. One set consisted of familiarity ratings measured mainly in 1995 in Kanto (i.e., metropolitan Tokyo and its suburbs), and they were registered in a word-familiarity database (Amano \& Kondo, 1999).

S.Amano, amano@cslab.kecl.ntt.co.jp 
The other set consisted of familiarity ratings measured in 2002 in Kinki (i.e., Osaka, Kyoto, Nara, and their suburbs), as described in the next section. These two places were selected because they have different dialects, which might affect the familiarity ratings. Two different years were chosen because we do not know whether familiarity ratings are consistent over a period of years. If familiarity rating is reliable for different years and places, the two sets would exhibit a high correlation coefficient.

\section{METHOD}

\section{Participants}

The participants $(N=40)$ were a mixed group consisting of members of the local community and undergraduate students recruited by a temporary employment agency. Half of the group were males and half were females. They all had at least a high school education. The participants were paid for participating in the experiment. The characteristics of the participants were almost the same as those in the familiarity rating experiment undertaken in 1995 in Kanto, for the development of the word-familiarity database (Amano \& Kondo, 1999). The main differences were that the participants in the present experiment were living in Kinki and had a Kinki dialect, whereas the participants in the 1995 experiment were living in Kanto and had a Kanto dialect. The average age of the participants was 23.1 $(S D=3.3$, $\max .=30, \min .=18)$, and their visual and hearing abilities were normal. To control the participants' language abilities, we employed the scores they obtained in the "Reading Ability Test for Kanji Words (100-Rakan)" (Kondo \& Amano, 1998, 2001). This test measures the ability of a person to read 100 kanji words, and it is designed to provide a score in the 0 to 100 range, where the average is about 50 . The score correlates highly with language abilities, including vocabulary size (Amano \& Kondo, 2000b) and readingaloud latency (Amano \& Kondo, 1999). The average 100-Rakan score of the participants was $66.4(S D=6.91$, max. $=85$, min. $=$ 60 ). A previous study (Kondo \& Amano, 2001) showed that the average score in the reading ability test was 55.1 and the $S D$ was 14.8 for a large number of samples (1,410 undergraduate students). Although the scores of almost all the present participants were within a range of two $S D$ s of the large number of samples, the average score of the present participants was higher than that of the large number of samples. Therefore, the present participants had a better than average language ability.

\section{Stimulus}

For the main test, 10,515 visual words were randomly selected from entries common to both the word-familiarity database (Amano \& Kondo, 1999; i.e., words with familiarity ratings obtained in 1995 in Kanto) and the Gakken Japanese dictionary (2nd ed., Kindaichi \& Ikeda, 1988). In addition to these words, 32,058 visual words were selected as filler words from the same dictionary. There were 42,573 words total. We used the large number of filler words because we wanted to extend the word-familiarity database by adding their familiarity ratings. The extended word-familiarity database will be published on a CD-ROM by the Sanseido publishing company in Tokyo (www.sanseido-publ.co.jp/) in the near future. The database will be called "Lexical Properties of Japanese, Volume 9."

For practice, 9,000 visual words were randomly selected from entries common both to the word-familiarity database and the Gakken Japanese dictionary. These words were different from the 10,515 test words. For a posttest, 3,000 visual words were randomly selected from the words in the main test.

\section{Procedure}

The procedure was the same as that of the 1995 familiarity rating experiment undertaken in Kanto to develop the word-familiarity database (Amano \& Kondo, 1999). The sole difference was that only visual words were used in the present experiment.
The participants were tested individually using a personal computer. The visual words were presented on the computer monitor using the standard set of Japanese characters. A different random order was used for the stimulus presentation for each participant. The participants were instructed to judge the familiarity of each presented word. They made judgments by using the computer mouse to click a single digit on a 7-point scale (1 for most unfamiliar to 7 for most familiar), which was displayed on the monitor of the personal computer. The participants were instructed to use the full range of the scale.

Practice was conducted with the 9,000 visual words. The participants were encouraged to develop their own rating criteria during the practice session, because no label or category for rating judgment was assigned to each number on the 7-point scale. The main test was conducted with the 42,573 visual words after the practice. A posttest to check the stability of the participants' familiarity rating was then performed with the 3,000 visual words, using the same procedure as that employed for the main test. The experiment was conducted for $3 \mathrm{~h}$ in the morning and $4 \mathrm{~h}$ in the afternoon, and lasted from February to March, 2002. The participants were allowed to take a 5-10-min break at any time during the experiment. The participants made approximately 4,000 judgments per day and took about 4 weeks to complete all the judgments.

\section{RESULTS AND DISCUSSION}

The correlation was $.949(N=10,515, p<.0001)$ between the mean familiarity ratings for the top $(n=20)$ and bottom $(n=20)$ halves of the present participant group based on their reading ability test scores. As in the 1995 familiarity rating experiment in Kanto, we set a condition whereby participants should have correlation coefficients higher than .5 between the main test and the posttest, with regard to the familiarity rating of visual words. Thirtyfour of 40 participants satisfied this condition. This number of participants was almost the same as that of the 1995 experiment. The participants' ratings were averaged for each word and used for the following analyses.

The mean familiarity rating for the visual test words was $4.11(N=10,515, S D=1.26, \max .=6.91, \min .=$ $1.09)$ in the present experiment, whereas the mean familiarity rating for these words was $4.19(N=10,515, S D=$ 1.36 , $\max .=6.75$, min. $=1.09)$ in the 1995 experiment. Although the difference between these means was significant $[t(10,514)=22.3, p<.0001$, two-tailed $]$, the values were very close. In addition, almost all the visual words had very similar familiarity ratings in both the 2002 and 1995 experiments, as shown in Figure 1. The correlation coefficient of the familiarity rating was extremely high $(r=.958, N=10,515)$.

The results indicate that the familiarity ratings for the 10,515 words selected from a dictionary are very consistent between the experiments. It is reasonable to conclude that the familiarity rating for ordinary words such as those found in a dictionary is reliable, even if the years and places differ.

The correlations between the familiarity rating and log frequency count $\left[\log _{10}(n+1)\right.$; Amano \& Kondo, 2000a] were $.691(N=10,515, p<.0001)$ for both the 2002 and 1995 experiments. The moderate correlation between familiarity and frequency count indicates that familiarity and frequency count have some discrepancy. The discrepancy is due to the fact that high-familiarity words 


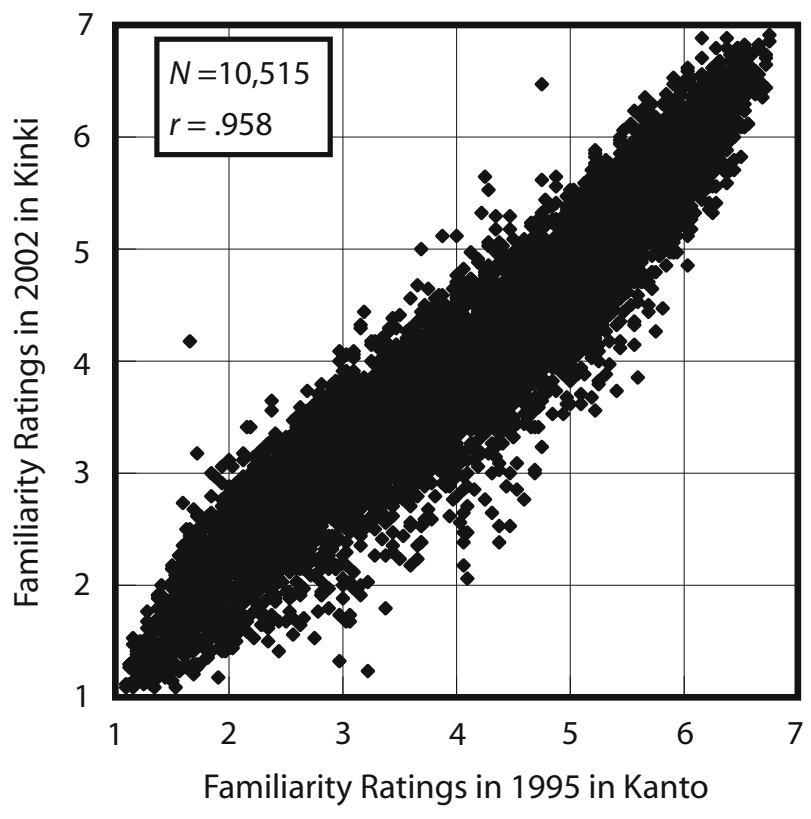

Figure 1. Scatterplot of familiarity ratings in 1995 in Kanto and in 2002 in Kinki.

do not frequently appear in a newspaper on which the frequency count is based. Such examples are misoshiru ("miso soup," familiarity $=6.38, \log$ frequency $=1.49$ ) and keshou-shitu ("bathroom," familiarity $=6.03$, log frequency $=1.51)$.

It is important to consider why very reliable familiarity ratings were obtained in the present and 1995 experiments despite the differences in participants, years, and places. One contributory factor is that the same procedure was used in both experiments. However, there are several other factors, as described below.

1. Preselection of participants. We selected participants based on their 100-Rakan score. This enabled us to choose participants with a certain uniformity of language ability, by selecting those whose ability was better. The preselection probably helped to reduce variability in the familiarity rating, because people with a better language ability know more words, so their familiarity rating would be more reliable.

2. Practice with many trials. The participants performed 9,000 practice trials. This large number of trials was designed to provide participants with an idea of the range of familiarity of a stimulus word set. It must also have given them an idea of which rating value on the 7-point scale appropriately represented their feeling of familiarity regarding a particular word. As a result, the participants became very good at providing a stable word familiarity rating.

3. Postselection of participants. We selected the participants according to correlation coefficients between the main and posttests. This enabled us to exclude participants with unstable familiarity ratings. To examine the effectiveness of this postselection, the familiarity ratings were recalculated for all participants $(N=40)$ and compared with those of the postselected participants $(n=34)$. For all the participants, the correlation coefficient between the present and the 1995 experiment was $957(N=10,515)$. This correlation coefficient is lower than that for the data obtained from the postselected participants. This indicates that participants with unstable familiarity ratings actually degrade the reliability. In other words, postselection was effective. However, the difference between the correlation coefficients (.001) is very small, thus showing that the postselection of participants is effective but does not contribute greatly to reliability.

This small effect might result from the fact that the number of unselected participants $(n=6)$ was much smaller than the number of selected participants $(n=34)$. If the number of unselected participants had been larger, the postselection would have had a greater effect. In this respect, we note that the number of selected participants must be an implicit factor with regard to reliable familiarity rating. The present results suggest that about 30 to 40 participants would be sufficient to provide reliable data if their rating skill were sufficiently stable. The numbers of participants in previous familiarity rating studies varied greatly. The present results suggest that in some cases, the numbers were too small (e.g., 11 [Amano et al., 1995], 12 [Nusbaum, Pisoni, \& Davis, 1984; Pisoni \& Garber, 1990], 15 [Kreuz, 1987], and 25 [Schulman, 1976]).

The factors described above must have contributed to the reliability of the data. However, we currently have no direct evidence to confirm the extent of this contribution, and further investigation is required.

Although this study offers clear results for the high reliability of the familiarity rating, we should be aware of certain limits, in terms of generalization.

First, the results are based on words derived from a dictionary. The stimulus word set included few proper nouns and few technical terms. The participants would probably exhibit less variation with regard to the words in a dictionary than with proper nouns or technical terms, because the former are commonly used in society. Hence, if proper nouns and technical terms were included in a stimulus word set, there might be greater variability in the familiarity rating. It is possible that the dictionary-based stimulus word set used here might have resulted in an overestimation of the reliability of the familiarity rating.

Second, the results are based solely on visual words. If auditory words were used for the experiment, the familiarity rating reliability might decrease. This is because the participants in the Kanto experiment had a Kanto dialect, whereas those in the Kinki experiment had a Kinki dialect, and these two dialects often have different lexical accent patterns for the same word. It remains an open question as to whether reliable familiarity ratings can be obtained for auditory words in different places with different dialects.

Third, only young adults participated in the experiments. We have no information to tell us whether familiarity rating is consistent for participants from other generations, such as children and elderly people. In addition, we do not yet know whether familiarity rating is consistent among generations. Further research over different generations will be necessary to clarify these points.

Fourth, only young adults with better than average language ability participated in the experiments. If participants with poor language ability participated in the exper- 
iments, the familiarity rating reliability might decrease. This idea is supported by the study of Auer, Bernstein, and Tucker (2000). They showed that the mean familiarity rating correlates with a participant's language ability, which indicates that familiarity ratings are different according to language ability of participants.

Fifth, participants of both experiments lived in urban areas. It is possible that the familiarity ratings are different between urban and rural areas. In that case, the familiarity rating reliability would decrease. But we have no information to tell us whether the familiarity ratings are consistent between places with different populations. This point should be clarified by further research.

Finally, the two experiments were conducted only about 7 years apart. This short period might have led to the high reliability of the familiarity rating. It is possible that the familiarity rating of each word changes over decades. However, we think that highly familiar words (e.g., taiyou ["sun"], haha ["mother"]) will remain highly familiar words in any era, because of their fundamental importance. Hence, highly familiar words, at least, will remain highly reliable with regard to their familiarity ratings over decades. However, from the present results, we can only say that the familiarity ratings of all words have almost the same values over about a half-decade.

\section{AUTHOR NOTE}

The authors thank Nozomu Inago and Tokomo Kanasugi for their assistance in conducting experiments and analyses. Correspondence concerning this article should be addressed to S. Amano, NTT Communication Science Laboratories, 2-4 Hikari-dai, Seika-cho, Souraku-gun, Kyoto 6190237 Japan (e-mail: amano@cslab.kecl.ntt.co.jp).

\section{REFERENCES}

Amano, S., \& Kondo, T. (1999). Nihongo-no Goi-tokusei [Lexical properties of Japanese] (Vols. 1-6). Tokyo: Sansei-do (in Japanese).

Amano, S., \& Kondo, T. (2000a). Nihongo-no Goi-tokusei [Lexical properties of Japanese] (Vol. 7). Tokyo: Sansei-do (in Japanese).

Amano, S., \& Kondo, T. (2000b). Relationship between vocabulary size and score of the reading ability test for kanji words. Proceedings of the 64th Annual Meeting of the Japanese Psychological Association (p. 520). Kyoto, Japan: Japanese Psychological Association (in Japanese)
Amano, S., Kondo, T., \& KaKehi, K. (1995). Modality dependency of familiarity ratings of Japanese words. Perception \& Psychophysics, 57, 598-603.

Auer, E. T., Bernstein, L. E., \& Tucker, P. E. (2000). Is subjective word familiarity a meter of ambient language? A natural experiment on effects of perceptual experience. Memory \& Cognition, 28, 789-797.

Connine, C. M., Mullennix, J., Shernoff, E., \& Yelen, J. (1990). Word familiarity and frequency in visual and auditory word recognition. Journal of Experimental Psychology: Learning, Memory, \& Cognition, 16, 1084-1096.

Kindaichi, H., \& IKeDA, Y. (EDS.) (1988). Gakken Kokugo-Daijiten [Gakken Large Japanese dictionary] (2nd ed.). Tokyo: GakushuKenshusya (in Japanese).

Kindaichi, K., Shibata, T., Yamada, A., \& Yamada, T. (Eds.) (1989). Shin-Meikai Kokugo Jiten [Shin-Meikai Japanese dictionary] (4th ed.). Tokyo: Sansei-do (in Japanese).

Kondo, T., \& Amano, S. (1998). The reading ability test for kanji words: An estimation method for language ability using word familiarity. Proceedings of the 62nd Annual Meeting of the Japanese Psychological Association (p. 711). Tokyo, Japan: Japanese Psychological Association (in Japanese).

Kondo, T., \& Amano, S. (2001). Score distribution of the reading ability test for kanji words "100-Rakan": What can be measured. Proceedings of the 65th Annual Meeting of the Japanese Psychological Association (p. 489). Tsukuba, Japan: Japanese Psychological Association (in Japanese).

KreUZ, R. J. (1987). The subjective familiarity of English homophones. Memory \& Cognition, 15, 154-168.

Lewellen, M. J., Goldinger, S. D., Pisoni, D. B., \& Greene, B. G. (1993). Lexical familiarity and processing efficiency: Individual differences in naming, lexical decision, and semantic categorization. Journal of Experimental Psychology: General, 122, 316-330.

Nusbaum, H. C., Pisoni, D. B., \& Davis, C. K. (1984). Sizing up the Hoosier mental lexicon: Measuring the familiarity of 20,000 words. Research on Speech Perception (Prog. Rep. No. 10, pp. 357-376). Bloomington: Indiana University, Speech Research Laboratory, Department of Psychology.

Pisoni, D. B., \& Garber, E. E. (1990). Lexical memory in visual and auditory modalities: The case for a common mental lexicon. Proceedings of the 1990 International Conference on Spoken Language Processing (pp. 401-404). Kobe: Acoustical Society of Japan.

Sakuma, N., IJuin, M. Fushimi, T., Tatsumi, I., Tanaka, M., Amano, S., \& Kondo, T. (2005). Nihongo-no Goi-tokusei [Lexical properties of Japanese] (Vol. 8). Tokyo: Sansei-do (in Japanese).

Schulman, A. I. (1976). Memory for rare words previously rated for familiarity. Journal of Experimental Psychology: Human Learning \& Memory, 2, 301-307.

(Manuscript received April 4, 2006; revision accepted for publication November 7, 2006.) 